\title{
When cells get stressed: an integrative view of cellular senescence
}

\author{
Ittai Ben-Porath and Robert A. Weinberg \\ The Whitehead Institute for Biomedical Research, Cambridge, Massachusetts, USA
}

\begin{abstract}
Cells entering a state of senescence undergo a permanent cell cycle arrest, accompanied by a set of functional and morphological changes. Senescence of cells occurs following an extended period of proliferation in culture or in response to various physiologic stresses, yet little is known about the role this phenomenon plays in vivo. The study of senescence has focused largely on its hypothesized role as a barrier to extended cell division, governed by a division-counting mechanism in the form of telomere length. Here, we discuss the biological functions of cellular senescence and suggest that it should be viewed in terms of its role as a general cellular stress response program, rather than strictly as a barrier to unlimited cycles of cell growth and division. We also discuss the relative roles played by telomere shortening and telomere uncapping in the induction of senescence.
\end{abstract}

J. Clin. Invest. 113:8-13 (2004). doi:10.1172/JCI200420663.

\section{Is senescence a biological program?}

Cellular senescence, one of the most fundamental aspects of cell behavior, was first described and formalized in the work of Hayflick in 1961 (1). Most types of primary normal cells that are grown in culture do not proliferate indefinitely. Instead, after a period of rapid proliferation, their division rate slows, ultimately ceasing altogether. Such cells become unresponsive to mitogenic stimuli yet can remain viable for extended periods of time. Upon entering the state of senescence, cells undergo a dramatic change in morphology - their volume increases and they lose their original shape, acquiring a flattened cytoplasm. This shift is accompanied by changes in nuclear structure, gene expression, protein processing, and metabolism (2-4). This form of senescence, which follows an extended period of propagation of cells in culture, has been termed "replicative senescence."

The study of the molecular mechanisms underlying senescence has shed light on central aspects of tumor development and has contributed to the research on organismal aging. Nonetheless, the role that cellular senescence itself plays in the living organism is still poorly understood. This stands in contrast to another

\footnotetext{
Address correspondence to: Robert A. Weinberg, The Whitehead Institute for Biomedical Research, 9 Cambridge Center,

Cambridge, Massachusetts 02142, USA. Phone: (617) 258-5159; Fax: (617) 258-5213; E-mail: weinberg@wi.mit.edu.

Conflict of interest: The authors have declared that no conflict of interest exists.

Nonstandard abbreviations used: human telomerase reverse transcriptase (hTERT); mouse embryo fibroblast (MEF); short interfering RNA (siRNA).
}

cellular response that serves to constrain cell proliferation - apoptosis - for which the molecular mechanisms and biological roles have been elucidated in minute detail over the past decade.

Can senescence be placed side-by-side with apoptosis as a fundamental biological program? Subsequent to Hayflick's discovery of replicative senescence, various studies have demonstrated that normal cells can undergo senescence rapidly in response to various physiologic stresses (5). This later work yielded a second category of senescence, often referred to as "stress-induced senescence." In fact, cells that are exposed to stress in culture will respond either by entry into senescence, by apoptosis, or by a transient growth arrest; the choice among these three responses depends on the cell type, the type of stress, and the level of stress. Hence, senescence seems to represent one of several programs that can be activated by the cell when physiologic stress is encountered.

The observation that a variety of stressors can bring about the senescence phenotype supports the notion that this phenotype represents a general cellular response mechanism rather than an idiosyncratic response to a specific type of physical or biochemical insult. Moreover, upon the activation of senescence, a plethora of changes in cellular morphology and function are induced in parallel, a task that is executed by specific molecular pathways.

These observations converge on the conclusion that senescence is a carefully orchestrated cellular program, indeed one that is likely to play an important role in the physiology of cells within living tissues. In truth, to date, only a handful of studies have reported the detection of senescence of cells in vivo (including recent contribu- 
tions described below). Thus, it is not clear that the functional and morphological changes that cells undergo upon entering senescence in culture also occur in vivo. Mouse mutants of the molecular activators of senescence $-p 53, R b$, and $p 16 /$ Ink $4 a$ - are tumor prone (6-9), suggesting that senescence serves as a tumor-suppressing mechanism. However, it has been difficult to directly demonstrate that it is the inactivation of the senescence program, rather than of other functions performed by these proteins, that leads to tumor development in these mice. Due to these difficulties, it remains possible that senescence is largely a phenomenon of cells growing in the culture dish and does not serve a physiologic role in vivo $(10,11)$.

However, two recent studies provide convincing demonstrations of senescence occurring in cells in vivo. In one of these studies, mice carrying eroded telomeres due to a mutant telomerase enzyme were subjected to partial hepatectomy (12). The hepatocytes of these mice displayed limited ability to proliferate and regenerate the liver; instead, they underwent senescence. In the other study, mice carrying lymphomas were treated by a chemotherapeutic agent (13). These lymphoma cells underwent senescence in vivo, but only when the apoptosis program was blocked by overexpression of the $b c l 2$ gene. Thus, given the proper experimental setting, senescence can clearly be observed in vivo, both in response to an internal signal - telomere attrition - and in response to an externally inflicted stress in the form of an alkylating agent. In both studies senescence was characterized by an arrest of division and by the appearance of senescence-associated $\beta$-galactosidase activity, a commonly used marker for senescence in vitro.

\section{A limit for proliferation, a response to stress, or both}

The initial discovery of the replicative senescence of human fibroblasts has led to the view that senescence serves as a mechanism whose purpose is to limit the proliferative capacity of normal cells $(11,14)$. According to this thinking, it is undesirable for cells to be capable of dividing beyond what is required for their participation in normal development and tissue maintenance. The capacity of cells to divide is therefore limited by an intrinsic mechanism that counts the number of divisions through which cell lineages have undergone, and triggers senescence when the predetermined limit for division is reached. While the senescence of cells might contribute to the aging of tissues, the breakdown of this division-restricting mechanism can lead to cancer.

The finding that telomeres, the nucleoprotein structures protecting chromosome ends, shorten with every cycle of cell growth and division suggested a molecular mechanism that could record the number of divisions that a lineage of cells has undergone. Erosion of telomeres to a critical length could serve to activate the senescence program (15). In accord with this mechanistic model, ectopic expression of the catalytic subunit of the telomerase enzyme, hTERT (human telomerase reverse transcriptase), halts the erosion of telomeres in human cells; in some cell types the expression of this gene prevents the entrance into replicative senescence, suggesting that indeed telomere shortening is the cause for senescence $(16,17)$.

As mentioned above, yet other work has demonstrated that normal cells that are exposed to various physiologic stresses rapidly enter into a state of senescence, doing so within a period as short as several days. Such stresses include DNA-damaging agents, oxidative stress, "oncogenic stress" (due to oncogene overexpression), and other metabolic perturbations (5, 18-22). Typically, these forms of senescence do not involve significant telomere shortening and cannot be prevented by ectopic hTERT expression $(23,24)$. Accordingly, the hypothesized telomere-based mechanism of division counting could not be invoked to explain these acute responses. Moreover, these situations of stress-induced senescence could not be accommodated by a model proposing that senescence functions exclusively as a barrier to extended growth-and-division cycles.

These observations raised the question of whether replicative senescence and stress-induced senescence serve the same biological function, and which of these mechanisms operates in vivo $(10,11,25)$. In fact, the conditions that induce these two responses are not as distinct as the above description would suggest. The onset of replicative senescence exhibited by some types of normal cells is dependent on the conditions in which they are propagated. This suggests that certain culture conditions are physiologically stressful to the cells, and that cells can undergo replicative senescence due to the cumulative effect of this stress, rather than the progressive erosion of their telomeres. For example, populations of human mammary epithelial cells encounter their first growth barrier following 10-20 divisions in culture. This stage of senescence can be avoided if these cells are grown on fibroblast feeder layers or in a different type of medium $(26,27)$. Similarly, mouse embryo fibroblasts (MEFs) senesce after approximately ten divisions. It was recently demonstrated that when MEFs are propagated in 3\% oxygen, rather than the commonly used $20 \%$ oxygen conditions, they can avoid senescence (28). This study also demonstrated that when grown in $20 \%$ oxygen, MEFs suffer from the rapid accumulation of DNA damage. Consequently, the cumulative oxidative damage induced by growth in conditions that are hyperoxic (by the standards of living tissues) leads to the onset of senescence in these cells. These findings provide a direct demonstration of how extrinsic physiologic stress experienced by cells can lead to replicative senescence.

In many cases, both types of mechanisms for the induction of replicative senescence - a telomere-based one and a stress-based one - seem to function together in the same cell population. Human fibroblasts are the beststudied example of a cell type in which the cause of senescence is attributed to critical telomere attrition, as their senescence can usually be prevented by ectopic expression of hTERT. However, these cells are hardly indifferent to 


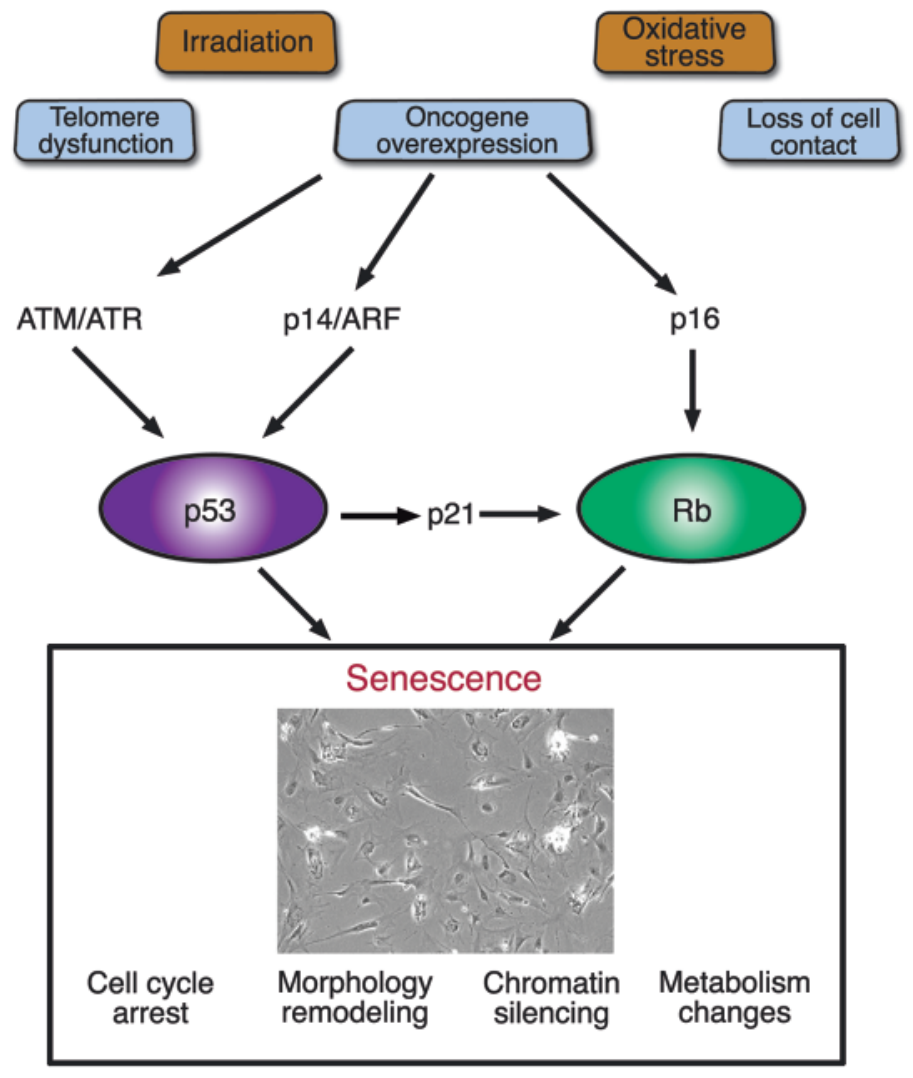

their growth conditions in vitro: the timing of their entry into senescence is affected by various parameters of culturing, such as plating density, media composition, and others. Thus, when propagated in 1-3\% oxygen instead of $20 \%$ oxygen, human fibroblasts are able to undergo more divisions prior to senescence; conversely, oxygen levels higher than $20 \%$ will shorten their lifespan (29-31). Clearly, a stress-based clock contributes to the effect of the telomere-based clock in these cells, and the combined effects of the two dictate the onset time of senescence.

Physiologic stress could hasten the onset of senescence in these cells in a manner independent of the process of telomere shortening. Alternatively, it could act by increasing the rates of telomere shortening. However, if the latter were true, then telomeric DNA could no longer be viewed as a counting device that advances autonomously at its own rate, but should rather be viewed as a cellular structure that responds to stress. In fact, it has been reported in the past that the telomeres of some cells shorten more quickly in high oxygen conditions (31). This observation received further substantiation in a recent study, demonstrating that in $2-5 \%$ oxygen conditions the rates of telomere shortening of some commonly studied human fibroblast lines - WI38 and IMR90 - are slower than the rates observed in 21\% oxygen (32). Moreover, when the hTERT gene is ectopically introduced into various human fibroblast lines, resulting in the elongation of their telomeres, the rate of telomere elongation is much slower in $21 \%$ oxygen than in $2-5 \%$ oxygen growth conditions. This study clearly

\section{Figure 1}

Senescence as a general stress-response program. A variety of physiologic stresses, intrinsic and extrinsic, lead to the onset of senescence. These stresses stimulate various cellular signaling pathways, which are funneled down to activate either the $\mathrm{p} 53$ protein, the $\mathrm{Rb}$ protein, or both. $\mathrm{p} 53$ can be activated by the DNA damage signaling pathways, the ATM and ATR proteins, or by the p14/ARF protein, which responds to oncogene overexpression and other stresses. p21, a target of p53, can cause the activation of Rb. Most cellular stresses will activate the $\mathrm{p} 16 / \mathrm{INK} 4 \mathrm{a}$ gene, also leading to $\mathrm{Rb}$ activation. Different stress signals will have different relative effects on the $\mathrm{p} 53$ and $\mathrm{Rb}$ arms, and their combined level of activation dictates the onset of senescence. Once this program is activated, a series of changes in cell function and morphology take place. ATM, ataxia telangiectasia mutated; ATR, ATM-related; p14/ARF, alternative reading frame product of INK4a gene locus.

demonstrates that telomere shortening is not an intrinsic clock-like mechanism that operates independently of extrinsic physiologic stresses. This recent study also underscores a little-regarded observation, namely that many normal human fibroblast lines cannot be immortalized by ectopic expression of hTERT. Telomerase activity enables these cells to proliferate longer, but eventually they do undergo senescence, even though their telomeres have been elongated well beyond the lengths observed in early passage cells (32). Thus, the immortalizing capabilities of telomerase are only limited to a subset of cell types.

Different types of intrinsic and extrinsic stress signals are likely to converge on the activation of the p 53 protein, the $\mathrm{Rb}$ protein, or both. In this manner, these two key tumor suppressor proteins might act as integrators of stress signals, and their combined level of activation would determine the onset of senescence (Figure 1). Recent studies have demonstrated that some cultured human fibroblast lines indeed express higher levels of the p16/INK4a gene than do others, presumably reflecting a higher degree of stress that these cells experience in vitro $(33,34)$. This stress-induced expression of $\mathrm{p} 16$, an activator of $\mathrm{Rb}$, apparently acts to hasten the replicative senescence of cells, doing so in a manner independent of telomere length $(34,35)$. These and other findings suggest that telomere attrition leads mainly to the activation of the p53 protein, while culture stresses mainly activate $R b$ through $p 16$.

The emerging picture is that various types of intrinsic and extrinsic stress stimuli can activate the senescence program, and whether this occurs rapidly, or gradually following a period of proliferation, is mainly determined by the combined levels of these stresses. Moreover, extrinsic signals may affect cell-intrinsic processes such as telomere shortening. It seems, then, that there is no necessity in a functional distinction between replicative senescence and stress-induced senescence, as these titles merely reflect the fact that a spectrum of different stimuli feed into one response program. 


\section{Telomeres - length or structure}

The telomeres of human cells that are propagated until they reach senescence erode, on average, to about half of their original length - from $10-20 \mathrm{~kb}$ to $5-10 \mathrm{~kb}$ in most cell types $(15,36)$. A situation of almost complete erosion of telomeres can be reached if senescence is bypassed through the inactivation the p53 and $\mathrm{Rb}$ pathways (37). When this is done, cells continue to divide beyond their normal senescence point, and their telomeres continue to shorten until a subsequent growth barrier, termed "crisis," is reached. At crisis, massive cell death occurs due to multiple chromosomal fusions and consequent genomic catastrophe. Senescence is thus induced at a midway point of telomere shortening. This provokes questions regarding the nature of the molecular changes that occur at the telomeres at this point, and the manner by which these changes activate the senescence program.

Different models have been proposed to explain how telomere shortening triggers senescence. Some models suggest that the onset of senescence is dictated by the length of the shortest telomere within a cell, rather than by the average length of telomeres (38). These models argue that due to variability in telomere lengths, there exists at least one telomere per cell that has eroded to a very short length at the time of senescence. Loss of telomeric repeats may cause the exposure of the chromosomal DNA end, which is recognized as a double-stranded break (39). Indeed, several studies have indicated that telomere lengths within a cell are highly variable, and that cells accumulate short telomeres as they approach senescence, some being as short as $2 \mathrm{~kb}$ or less $(40,41)$.

Other studies have argued, however, that the relationship between telomere length and senescence is more complex. Telomere length does not always strictly correlate with the induction of senescence, and it is difficult to point to a consistent critical length that induces the senescence response $(42,43)$. Some cell lines are capable of dividing with very short telomeres, a situation that is apparently sustained by ongoing telomerase expression (44). It has been recently shown that human fibroblasts that overexpress the telomerebinding protein TRF2 display an accelerated rate of telomere shortening; surprisingly, these cells undergo senescence following the same number of divisions as control cells, even though their telomeres at senescence are shorter (45). This study clearly demonstrates that, at least in the case of these cells, it is not the length of the double-stranded region per se that dictates the timing of senescence.

Many groups have therefore arrived at the hypothesis that it is probably not the actual telomere length - the number of double-stranded hexameric repeat units at the telomeres - that is the molecular feature directly dictating the timing of senescence. Instead, the structure of telomeres - the nucleoprotein complex that serves as a protective "cap" for the chromosome end may undergo changes during extended cell prolifera- tion, possibly as a result of telomere shortening. "Uncapping" of telomeres, rather than shortening of telomeres, may, according to this thinking, lead to the exposure of the chromosome ends and to the activation of the senescence program $(42,46)$.

\section{Telomere uncapping at senescence}

Relatively little is known about the structure that is formed by the DNA and protein components of the telomeric cap. The double-stranded stretch of hexameric repeats that comprises several kilobases of telomeric DNA ends with a $3^{\prime}$ overhang of 100-400 nucleotides (47-49) (Figure 2a). This overhang is considered to be a critical component of telomere structure, and appears to
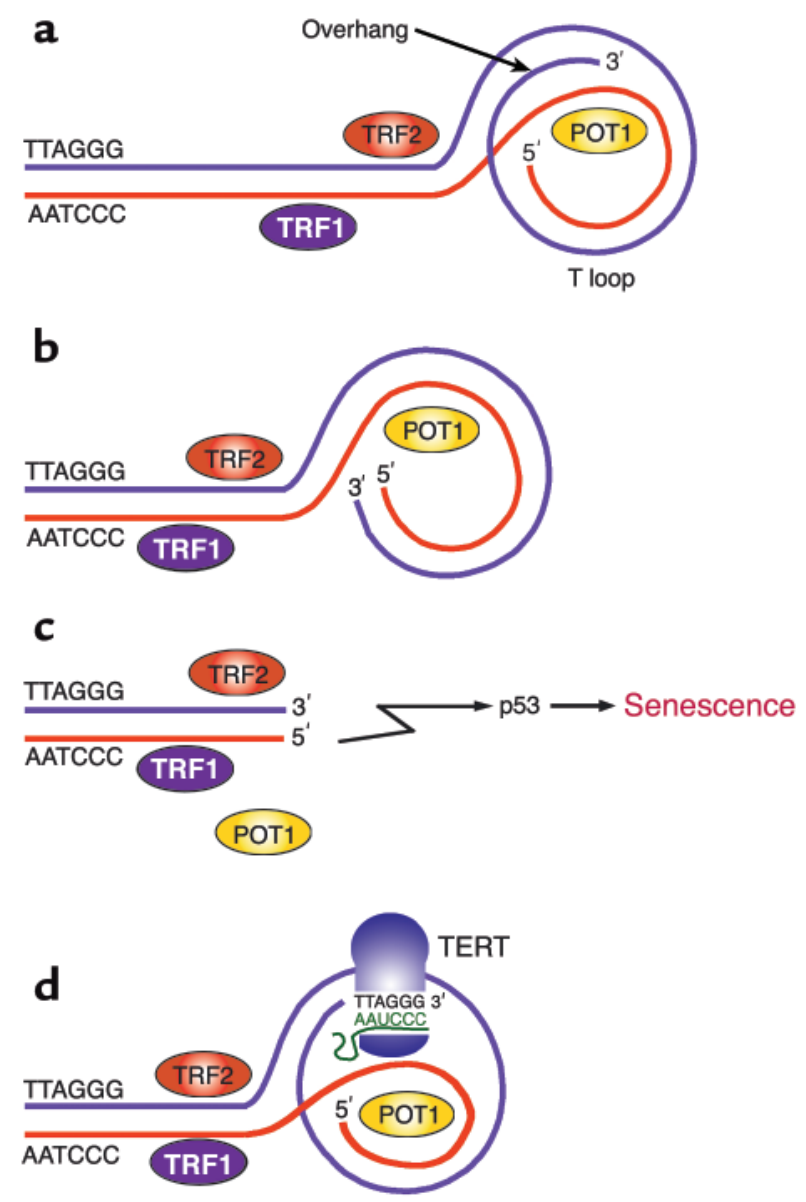

\section{Figure 2}

Telomere uncapping at senescence. The nucleoprotein structure at the end of telomeres presumably forms a protective cap. This structure may be composed of the T-loop (a), which is formed by the invasion of the single-stranded overhang into an upstream doublestranded region of the telomere, and of protective telomere-binding proteins such as TRF1, TRF2, and POT1. As cells approach replicative senescence, the double-stranded portion of the telomere shortens, and the single-stranded overhang is eroded (b). This may cause the collapse of the telomere cap and the exposure of the telomere end, which is detected by the DNA damage machinery and leads to the activation of the senescence program (c). Telomerase activity, apart from stabilizing overall telomere length, can prevent overhang erosion and protect the telomere cap, thereby circumventing senescence (d). TERT, telomerase enzyme reverse transcriprase. 
be actively generated on every telomere following DNA replication. It has been shown that the ends of telomeric DNA can exist in a structure termed the "T-loop," which is formed by the invasion of this single-stranded overhang into an upstream double-stranded region of telomeric DNA (50). Such a second-order DNA structure, bound and held together by specific telomeric proteins, notably TRF1, TRF2, and POT1, appears to function as the protective cap for the telomere.

Do telomeres undergo uncapping at senescence? The lack of direct molecular markers for the capped or uncapped state of a telomere has limited the ability to address this question. The detection of the T-loop structure itself is technically extremely challenging, and does not easily facilitate quantitative assessment of T-loop numbers in different cells. The telomeric overhang, however, being a putative key component of telomere structure, could well serve as an indicator of the telomere capping state. The ability to assess overhang lengths has been facilitated by a recently developed technique - the telomeric oligonucleotide ligation assay (T-OLA) (51). Use of this assay has revealed that in senescent cells the telomeric overhang is significantly eroded (Figure 2, b and c), when compared to overhang lengths in early-passage cells (52). The extent of overhang erosion suggests that a large proportion of the telomeres in a cell have lost much of their singlestranded overhang DNA, even though most of these telomeres still carry a significant number of repeats in their double-stranded portion.

It is reasonable to assume that telomeres that have lost their single-stranded overhangs are functionally uncapped. Such telomeres may have lost their ability to form T-loops, and in addition may have lost the protection afforded by certain telomeric proteins. This process may lead to exposure of the chromosome end, activation of the DNA damage machinery (39), and the triggering of senescence (Figure 2c). The fact that telomere uncapping can lead to senescence has been demonstrated experimentally. Expression of a dominant-negative form of the TRF2 protein causes loss of the telomeric overhang and the formation of telomeric fusions, without loss of overall telomere length in the doublestranded region $(53,54)$. When this protein is expressed in normal fibroblasts, the result is the induction of rapid senescence, demonstrating that the forced uncapping of telomeres activates the senescence program (55).

On the basis of these findings, it can be argued that as cells approach senescence, the physical structure of telomeres undergoes changes that result in the loss of protective capping. Perhaps the most central question regarding this process is whether telomere uncapping is a consequence of telomere shortening, or whether it is an event that occurs independently of overall telomere length, being induced by other cellular signals.

\section{Telomerase as a cap protector}

More light on the role played by the telomere cap in senescence has been shed by recent discoveries regard- ing the function of the telomerase enzyme. Introduction of the hTERT catalytic subunit into presenescent fibroblasts results in an elongation of the doublestranded region of the telomere $(16,17)$. The recent examination of the telomeric overhang in such cells has revealed that ectopic hTERT expression also protects the telomeric overhang from erosion, and can, in fact, elongate it (52) (Figure 2d). This finding is not surprising, since the direct biochemical activity of telomerase is to add repeat units to the $3^{\prime}$ end of the telomere, i.e. to the overhang, whereas the extension of the double-stranded region occurs only upon complementation of the elongated overhang, possibly by conventional DNA polymerases. By protecting overhang DNA from erosion, telomerase activity can maintain proper telomere structure. This raises the possibility that it is this aspect of telomerase activity, and not the elongation of the double stranded telomere region, which is critical for the prevention of senescence (Figure 2d).

Yet another recent study indicates that maintenance of proper telomere structure is in fact performed by the endogenous telomerase enzyme, operating in presenescent normal cells (56). It has long been believed that most normal human cells do not express the bTERT gene and therefore do not possess telomerase activity. This recent work has shown, using sensitive detection methods, that $b T E R T$ is in fact expressed and active in normal human fibroblasts. This expression is transient, appearing only during S-phase (56). When this transient endogenous hTERT expression was eliminated in these cells through the use of a small interfering RNA (siRNA) vector, the cells underwent premature replicative senescence. Strikingly, the rate of overall telomere shortening did not change in these cells. Instead, their telomeric overhangs eroded more rapidly than did those of control cells, so that fibroblasts expressing the siRNA against hTERT and entering senescence carried significantly eroded overhangs, whereas control cells that underwent the same number of divisions carried overhangs of normal length (56).

These findings hold several important implications. First, they demonstrate that it is possible to accelerate the erosion of telomeric overhangs (and attendant telomere uncapping) without affecting overall telomere shortening rates. Second, they indicate that it is the capping state of telomeres that correlates with the induction of senescence, and not the overall length. Third, they point to a role for the telomerase enzyme in normal cells - maintenance of telomere structure. Conceivably, the S-phase-specific activity of telomerase serves to maintain the properly capped state of telomeres following DNA replication, apparently without preventing the progressive erosion of overall telomere length that occurs during the cycles of growth and division of normal human cells.

\section{Conclusions}

The contribution of the senescence program to the physiology of living tissues, to aging processes, to 
disease, and to the prevention of tumor development remains poorly understood. As discussed above, it is not clear that the central role of this program is the limitation of the division capacity of cells. Rather, its role as a general response to physiologic stress seems increasingly important. Moreover, the postulated function of telomeres as a counting mechanism for the number of cell divisions, which acts to activate senescence after a predetermined number of such divisions, should be reviewed. It seems that even in the cases where telomeres play a prominent role in the induction of senescence, it is questionable whether they can be regarded simply as division-counting devices.

\section{Acknowledgments}

The authors would like to thank Kimberly Hartwell for reviewing of the manuscript. Ittai Ben-Porath is supported by an Anna Fuller Fund postdoctoral fellowship.

1. Hayflick, L., and Moorhead, P.S. 1961. The serial cultivation of human diploid cell strains. Exp. Cell Res. 25:585-621.

2. Campisi, J. 2000. Cancer, aging and cellular senescence. In Vivo. 14:183-188.

3. Sitte, N., Merker, K., Von Zglinicki, T., Grune, T., and Davies, K.J. 2000. Protein oxidation and degradation during cellular senescence of human BJ fibroblasts. I. Effects of proliferative senescence. FASEB J. 14:2495-2502.

4. Narita, M., et al. 2003. Rb-mediated heterochromatin formation and silencing of E2F target genes during cellular senescence. Cell. 113:703-716.

5. Lloyd, A.C. 2002. Limits to lifespan. Nat. Cell Biol. 4:E25-E27.

6. Donehower, L.A., et al. 1992. Mice deficient for p53 are developmentally normal but susceptible to spontaneous tumours. Nature. 356:215-221.

7. Jacks, T., et al. 1992. Effects of an Rb mutation in the mouse. Nature. 359:295-300.

8. Krimpenfort, P., Quon, K.C., Mooi, W.J., Loonstra, A., and Berns, A. 2001. Loss of p16Ink4a confers susceptibility to metastatic melanoma in mice. Nature. 413:83-86.

9. Sharpless, N.E., et al. 2001. Loss of p16Ink4a with retention of p19Arf predisposes mice to tumorigenesis. Nature. 413:86-91.

10. Sherr, C.J., and DePinho, R.A. 2000. Cellular senescence: mitotic clock or culture shock? Cell. 102:407-410.

11. Wright, W.E., and Shay, J.W. 2002. Historical claims and current interpretations of replicative aging. Nat. Biotechnol. 20:682-688.

12. Satyanarayana, A., et al. 2003. Telomere shortening impairs organ regeneration by inhibiting cell cycle re-entry of a subpopulation of cells. EMBOJ. 22:4003-4013.

13. Schmitt, C.A., et al. 2002. A senescence program controlled by p53 and p16INK4a contributes to the outcome of cancer therapy. Cell. 109:335-346.

14. Hayflick, L. 1998. How and why we age. Exp. Gerontol. 33:639-653.

15. Harley, C.B., Futcher, A.B., and Greider, C.W. 1990. Telomeres shorten during ageing of human fibroblasts. Nature. 345:458-460.

16. Bodnar, A.G., et al. 1998. Extension of life-span by introduction of telomerase into normal human cells. Science. 279:349-352.

17. Vaziri, H., and Benchimol, S. 1998. Reconstitution of telomerase activity in normal human cells leads to elongation of telomeres and extended replicative life span. Curr. Biol. 8:279-282.

18. Fairweather, D.S., Fox, M., and Margison, G.P. 1987. The in vitro lifespan of MRC-5 cells is shortened by 5-azacytidine-induced demethylation. Exp. Cell Res. 168:153-159.

19. Chen, Q., and Ames, B.N. 1994. Senescence-like growth arrest induced by hydrogen peroxide in human diploid fibroblast F65 cells. Proc. Natl. Acad. Sci. U. S. A. 91:4130-4134.

20. Venable, M.E., Lee, J.Y., Smyth, M.J., Bielawska, A., and Obeid, L.M. 1995. Role of ceramide in cellular senescence. J. Biol. Chem. 270:30701-30708.

21. Serrano, M., Lin, A.W., McCurrach, M.E., Beach, D., and Lowe, S.W. 1997. Oncogenic ras provokes premature cell senescence associated with accumulation of p53 and p16INK4a. Cell. 88:593-602.

22. Robles, S.J., and Adami, G.R. 1998. Agents that cause DNA double strand breaks lead to p16INK4a enrichment and the premature senescence of normal fibroblasts. Oncogene. 16:1113-1123.

23. Wei, S., and Sedivy, J.M. 1999. Expression of catalytically active telomerase does not prevent premature senescence caused by overexpression of oncogenic Ha-Ras in normal human fibroblasts. Cancer Res. 59:1539-1543.
24. Gorbunova, V., Seluanov, A., and Pereira-Smith, O.M. 2002. Expression of human telomerase (hTERT) does not prevent stress-induced senescence in normal human fibroblasts but protects the cells from stressinduced apoptosis and necrosis. J. Biol. Chem. 277:38540-38549.

25. Wright, W.E., and Shay, J.W. 2000. Telomere dynamics in cancer progression and prevention: fundamental differences in human and mouse telomere biology. Nat. Med. 6:849-851.

26. Ramirez, R.D., et al. 2001. Putative telomere-independent mechanisms of replicative aging reflect inadequate growth conditions. Genes Dev. 15:398-403.

27. Herbert, B.S., Wright, W.E., and Shay, J.W. 2002. p16(INK4a) inactivation is not required to immortalize human mammary epithelial cells. Oncogene. 21:7897-7900.

28. Parrinello, S., et al. 2003. Oxygen sensitivity severely limits the replicative lifespan of murine fibroblasts. Nat. Cell Biol. 5:741-747.

29. Packer, L., and Fuehr, K. 1977. Low oxygen concentration extends the lifespan of cultured human diploid cells. Nature. 267:423-425.

30. Chen, Q., Fischer, A., Reagan, J.D., Yan, L.J., and Ames, B.N. 1995. Oxidative DNA damage and senescence of human diploid fibroblast cells. Proc. Natl. Acad. Sci. U. S. A. 92:4337-4341.

31. von Zglinicki, T., Saretzki, G., Docke, W., and Lotze, C. 1995. Mild hyperoxia shortens telomeres and inhibits proliferation of fibroblasts: a model for senescence? Exp. Cell Res. 220:186-193.

32. Forsyth, N.R., Evans, A.P., Shay, J.W., and Wright, W.E. 2003. Developmental differences in the immortalization of lung fibroblasts by telomerase. Aging Cell. 2:235-243.

33. Beausejour, C.M., et al. 2003. Reversal of human cellular senescence: roles of the p53 and p16 pathways. EMBOJ. 22:4212-4222.

34. Itahana, K., et al. 2003. Control of the replicative life span of human fibroblasts by 16 and the polycomb protein Bmi-1. Mol. Cell. Biol. 23:389-401.

35. Wei, W., Herbig, U., Wei, S., Dutriaux, A., and Sedivy, J.M. 2003. Loss of retinoblastoma but not $\mathrm{p} 16$ function allows bypass of replicative senescence in human fibroblasts. EMBO Rep. 4:1061-1065.

36. Allsopp, R.C., and Harley, C.B. 1995. Evidence for a critical telomere length in senescent human fibroblasts. Exp. Cell Res. 219:130-136.

37. Shay, J.W., Pereira-Smith, O.M., and Wright, W.E. 1991. A role for both $\mathrm{RB}$ and $\mathrm{p} 53$ in the regulation of human cellular senescence. Exp. Cell Res. 196:33-39.

38. Harley, C.B., Vaziri, H., Counter, C.M., and Allsopp, R.C. 1992. The telomere hypothesis of cellular aging. Exp. Gerontol. 27:375-382.

39. d'Adda di Fagagna, F., et al. 2003. A DNA damage checkpoint response in telomere-initiated senescence. Nature. 426:194-198.

40. Martens, U.M., Chavez, E.A., Poon, S.S., Schmoor, C., and Lansdorp, P.M. 2000. Accumulation of short telomeres in human fibroblasts prior to replicative senescence. Exp. Cell Res. 256:291-299.

41. Baird, D.M., Rowson, J., Wynford-Thomas, D., and Kipling D. 2003. Extensive allelic variation and ultrashort telomeres in senescent human cells. Nat. Genet. 33:203-207.

42. Blackburn, E.H. 2000. Telomere states and cell fates. Nature. 408:53-56

43. Rubin, H. 2002. The disparity between human cell senescence in vitro and lifelong replication in vivo. Nat. Biotechnol. 20:675-681.

44. Zhu, J., Wang, H., Bishop, J.M., and Blackburn, E.H. 1999. Telomerase extends the lifespan of virus-transformed human cells without net telomere lengthening. Proc. Natl. Acad. Sci. U. S. A. 96:3723-3728.

45. Karlseder, J., Smogorzewska, A., and de Lange, T. 2002. Senescence induced by altered telomere state, not telomere loss. Science. 295:2446-2449.

46. Blackburn, E.H. 2001. Switching and signaling at the telomere. Cell. 106:661-673.

47. McElligott, R., and Wellinger, R.J. 1997. The terminal DNA structure of mammalian chromosomes. EMBOJ. 16:3705-3714

48. Wellinger, R.J., and Sen, D. 1997. The DNA structures at the ends of eukaryotic chromosomes. Eur. J. Cancer. 33:735-749.

49. Wright, W.E., Tesmer, V.M., Huffman, K.E., Levene, S.D., and Shay, J.W. 1997. Normal human chromosomes have long G-rich telomeric overhangs at one end. Genes Dev. 11:2801-2809.

50. Griffith, J.D., et al. 1999. Mammalian telomeres end in a large duplex loop. Cell. 97:503-514.

51. Cimino-Reale, G., et al. 2001. The length of telomeric G-rich strand 3 '-overhang measured by oligonucleotide ligation assay. Nucleic Acids Res. 29:E35.

52. Stewart, S.A., et al. 2003. Erosion of the telomeric single-strand overhang at replicative senescence. Nat. Genet. 33:492-496.

53. van Steensel, B., Smogorzewska, A., and de Lange, T. 1998. TRF2 protects human telomeres from end-to-end fusions. Cell. 92:401-413.

54. Takai, H., Smogorzewska, A., and de Lange, T. 2003. DNA damage foci at dysfunctional telomeres. Curr. Biol. 13:1549-1556.

55. Smogorzewska, A., and de Lange, T. 2002. Different telomere damage signaling pathways in human and mouse cells. EMBO J. 21:4338-4348.

56. Masutomi, K., et al. 2003. Telomerase maintains telomere structure in normal human cells. Cell. 114:241-253. 